\title{
MINIMAL REGULARITY CONDITIONS FOR THE END-POINT ESTIMATE OF BILINEAR CALDERÓN-ZYGMUND OPERATORS
}

\author{
CARLOS PÉREZ AND RODOLFO H. TORRES
}

(Communicated by Alexander Iosevich)

Dedicated to the memory of Cora Sadosky

\begin{abstract}
Minimal regularity conditions on the kernels of bilinear operators are identified and shown to be sufficient for the existence of end-point estimates within the context of the bilinear Calderón-Zygmund theory.
\end{abstract}

\section{INTRODUCTION}

A crucial property addressed in the linear Calderón-Zygmund theory, going back to the founding article [1, is the fact that operators bounded on $L^{2}$ whose kernels possess certain regularity are in fact bounded on every $L^{p}$ space for $1<p<\infty$. Moreover, such a regularity assumption implies, together with the $L^{2}$-boundedness of the operator, a weak-type end-point estimate in $L^{1}$. From these continuity properties, the whole range of values of $p$ follows by duality and interpolation. The quest for the minimal amount of regularity needed to guarantee the existence of such an end-point estimate has a rich history, with several important results that promoted as a byproduct numerous developments in harmonic analysis. For classical singular integrals operators with homogeneous kernels, the question was finally settled in the work of Seeger [23], who showed that the kernel of the operators could be quite rough. See also previous work of Christ [5] and Christ and Rubio de Francia [7]. We refer to [23] and its featured review by Hofmann [13] for precise technical details, an account of the history, and relevant references. For more general multiplier operators, as well as operators of non-convolution type, the regularity of the kernel is very closely related to a Lipschitz-type one. It is convenient for our purposes to recall some well-known facts related to this.

Assume that $T: L^{2}\left(\mathbb{R}^{n}\right) \rightarrow L^{2}\left(\mathbb{R}^{n}\right)$ is an operator that, at least for $x \notin \operatorname{supp} f$, is given by

$$
T f(x)=\int K(x, y) f(y) d y .
$$

Here, the kernel $K$ is assumed to be an integrable function on any product $E_{1} \times E_{2}$ of compact sets in $\mathbb{R}^{n}$, with $E_{1} \cap E_{2}=\emptyset$. Then, a sufficient condition for the

Received by the editors May 29, 2012.

2010 Mathematics Subject Classification. Primary 42B20, 42B25.

Key words and phrases. Multilinear singular integrals, Calderón-Zygmund theory, weak-type estimates, end-point estimates.

The first author's research was supported in part by the Spanish Ministry of Science and Innovation grant MTM2009-08934 and by the Junta de Andalucía grant FQM-4745.

The second author's research was supported in part by the National Science Foundation under grant DMS 1069015. 
operator to satisfy $T: L^{1} \rightarrow L^{1, \infty}$ is the gradient condition on the kernel given by

$$
\left|\nabla_{y} K(x, y)\right| \lesssim \frac{1}{|x-y|^{n+1}} \text { for } x \neq y
$$

Alternatively one can assume a Lipschitz form of the above,

$$
\left|K(x, y)-K\left(x, y^{\prime}\right)\right| \lesssim \frac{\left|y-y^{\prime}\right|^{\epsilon}}{|x-y|^{n+\epsilon}} \text { for }\left|y-y^{\prime}\right| \leq c|x-y|,
$$

for some $0<\epsilon \leq 1$ and $0<c<1$. Moreover, one can also consider a weaker condition which takes the form

$$
\int_{\left|y-y^{\prime}\right| \leq c|x-y|}\left|K(x, y)-K\left(x, y^{\prime}\right)\right| d x<\infty .
$$

This is sometimes referred to as the integral regularity or Hörmander condition. This condition is very convenient when working on more general geometric or measure theoretic contexts where other types of regularity are absent or hard to formalize. Actually, in $\mathbb{R}^{n}$ it can be written in the more geometric form

$$
\sup _{Q} \sup _{y \in Q} \int_{\mathbb{R}^{n} \backslash Q^{*}}\left|K(x, y)-K\left(x, y_{Q}\right)\right| d x<\infty,
$$

where the supremum is taken over all cubes $Q$ in $\mathbb{R}^{n}$ with sides parallel to the axes, and where $Q^{*}$ is the cube with the same center $y_{Q}$ as $Q$ and side length an appropriately chosen large multiple $c_{n}$ of the side length of $Q$ (for example $c_{n}=10 \sqrt{n} \ell(Q)$ will do).

To some extent, condition (1.3) is barely enough to prove the end-point estimate $L^{1} \rightarrow L^{1, \infty}$ but, unlike (1.1) or (1.2), it is not enough for other aspects of the Calderón-Zygmund theory. In particular it does not suffice to establish good- $\lambda$ inequalities between $T$ and the Hardy-Littlewood maximal function $M$, and there is no weighted theory for operators satisfying only (1.3); see the work of MartellPérez-Trujillo 20 .

We also recall that in the case of multiplier operators, that is, kernels of the form $K(x-y)$, (1.3) follows from the Hörmander-Mihlin conditions. Let $T$ be given by

$$
\widehat{T f}(\xi)=m(\xi) \widehat{f}(\xi) .
$$

Then, both the Mihlin [21] condition

$$
\left|\partial^{\alpha} m(\xi)\right| \lesssim|\xi|^{-|\alpha|} \text { for all }|\alpha| \leq[n / 2]+1
$$

and the weaker Hörmander [14] condition

$$
\sup _{j \in \mathbb{Z}}\left\|m\left(2^{j} \cdot\right) \varphi\right\|_{L_{s}^{2}\left(\mathbb{R}^{n}\right)}<\infty \text { for some } s>n / 2,
$$

where $\varphi$ is a smooth bump supported away from the origin and $L_{s}^{2}$ is the usual Sobolev space, imply (1.3).

We now describe the situation of the bilinear Calderón-Zygmund theory. Under regularity assumptions analogous to (1.1) or (1.2), the theory has been developed through works of Coifman-Meyer [2, 3], 4], Christ-Journé 6], Kenig-Stein [15] and Grafakos-Torres [10, [1]. Some of the most recent work in the subject was motivated in part by the results of Lacey-Thiele [16], [17] on the bilinear Hilbert transform and a search for the optimal range of exponents where boundedness in Lebesgue spaces can be obtained. Unlike the case of the bilinear Hilbert transform, 
a more singular operator not covered by the Calderón-Zygmund theory, the boundeness of Calderón-Zygmund operators on the full range of exponents is known. In particular, it was shown in [1] that a bilinear operator bounded from $L^{p} \times L^{q} \rightarrow L^{r}$ for some $1 / p+1 / q=1 / r$, and given by

$$
T(f, g)(x)=\int_{\mathbb{R}^{2 n}} K(x, y, z) f(y) g(z) d y d z
$$

for $x \notin \operatorname{supp} f \cap \operatorname{supp} g$, also satisfies $T: L^{1} \times L^{1} \rightarrow L^{1 / 2, \infty}$, provided the Schwartz kernel of the operator has the Lipschitz regularity properties

$$
\left|K(x, y, z)-K\left(x, y^{\prime}, z\right)\right| \lesssim \frac{\left|y-y^{\prime}\right|^{\epsilon}}{(|x-y|+|x-z|)^{n+\epsilon}}
$$

whenever $\left|y-y^{\prime}\right| \leq \frac{1}{2} \max \{|x-y|,|x-z|\}$ and

$$
\left|K(x, y, z)-K\left(x, y, z^{\prime}\right)\right| \lesssim \frac{\left|z-z^{\prime}\right|^{\epsilon}}{(|x-y|+|x-z|)^{n+\epsilon}}
$$

whenever $\left|z-z^{\prime}\right| \leq \frac{1}{2} \max \{|x-y|,|x-z|\}$. As in the linear theory, if $T$ and its two transposes $T^{* 1}$ and $T^{* 2}$ satisfy the same conditions, then interpolation and duality give boundedness for the full range of exponents, $L^{p} \times L^{q} \rightarrow L^{r}$ for all $1 / p+1 / q=1 / r$ and $1 / 2<r<\infty$. Moreover, the regularity assumptions are also good enough to obtain a weighted theory for classical $A_{p}$ weights, as shown by Grafakos-Torres [12] and Pérez-Torres 22, which was later extended to new optimal multilinear classes of weights by Lerner-Ombrosi-Pérez-Torres-Trujillo [18.

There has been, after all of these works, some interest in finding the corresponding analog to the minimal regularity assumption (1.3) in the multilinear setting. Our purpose with this short note is to contribute in this regard.

We note that Maldonado and Naibo [19] have weakened (1.7) and (1.8) to a Diny-type condition that we shall also consider (see Definition 2.2 below), and they simplified the proof of the end-point estimate. However, we are able in this article to identify integral type conditions which are even weaker and to further simplify the proof of the $L^{1} \times L^{1} \rightarrow L^{1 / 2, \infty}$ result. We focus only on the bilinear setting, but the interested reader may find analogous conditions in the $m$-linear case. The reader may also proceed to the next section, where we state the conditions, but we want to provide some heuristic considerations that led to the conditions we use. To do so, we recall very recent results for bilinear multiplier operators (corresponding to operators with kernels of the form $K(x-y, x-z)$ ). It is of interest that the parallel to the linear theory in terms of minimal regularity for multipliers appears to break down.

Suppose that $T_{m}$ is a Coifman-Meyer bilinear multiplier operator. That is,

$$
T_{m}(f, g)(x)=\int m(\xi, \eta) \widehat{f}(\xi) \widehat{g}(\eta) e^{i x(\xi+\eta)} d \xi d \eta
$$

where

$$
\left|\partial_{\alpha} m(\xi, \eta)\right| \lesssim_{\alpha}(1+|\xi|+|\eta|)^{-|\alpha|} \text { for all } \alpha .
$$

Then the kernel of $T_{m}$ is of the form $K(x-y, x-z)$ and satisfies

$$
\left|\partial_{\alpha} K(x-y, x-z)\right| \lesssim \alpha \frac{1}{(|x-y|+|x-z|)^{2 n+|\alpha|}} .
$$


This follows from considering $m$ as a multiplier in $\mathbb{R}^{2 n}$ and restricting the corresponding kernel $K\left(x_{1}-y, x_{2}-z\right)$ to $x_{1}=x_{2}$. It also follows then that these operators are bounded from $L^{p} \times L^{q} \rightarrow L^{r}$ for the full range of exponents and also from $L^{1} \times L^{1}$ to $L^{1 / 2, \infty}$.

It is very natural to expect that (1.9) could be relaxed to a Hörmander-Mihlin condition, limiting the number of derivatives of the symbol needed to be controlled. Certainly the arguments used by Coifman-Meyer only need a "sufficiently large" number of derivatives (not easy to track in their computations), but one should expect to require only about "half-the-dimension" number of derivatives. However, the natural dimension in the bilinear setting appears to be $2 n$, and so some interesting situations occur.

In fact, Tomita 24] recently showed that

$$
\sup _{j \in \mathbb{Z}}\left\|m\left(2^{j} \cdot, 2^{j} \cdot\right) \varphi\right\|_{L_{s}^{2}\left(\mathbb{R}^{2 n}\right)}<\infty \text { for some } s>n,
$$

where $\varphi$ is an appropriate cut-off function in $\mathbb{R}^{2 n}$, implies the boundedness

$$
T_{m}: L^{p} \times L^{q} \rightarrow L^{r}
$$

with $1 / p+1 / q=1 / r$, but only for $r>1$, that is, essentially "half-the-dimension" number of derivatives in $L^{2}$ to obtain the range $r>1$. However, to obtain other values of $1 / 2<r \leq 1$, which is also natural in the bilinear case, it appears that one needs to impose higher regularity. Grafakos-Si [9] showed after Tomita's work that one can push $p, q$ to $1+\epsilon$ (i.e. $r$ to $1 / 2+\epsilon / 2$ ) if

$$
\sup _{j \in \mathbb{Z}}\left\|m\left(2^{j} \cdot, 2^{j} \cdot\right) \varphi\right\|_{L_{s}^{t_{\epsilon}\left(\mathbb{R}^{2 n}\right)}}<\infty
$$

for appropriate $1<t_{\epsilon} \leq 2$ and $s>2 n / t_{\epsilon}$. Essentially, one may say that $2 n$ derivatives in $L^{1}$ may be required to get the full range of exponents. We refer to [9] for the precise technical details. Similar results on the product of Hardy $H^{p}$ were very recently obtained by Grafakos-Miyachi-Tomita [8], but, as far as we know and unlike the linear case, there are no results of this type that give the end-point estimate $L^{1} \times L^{1} \rightarrow L^{1 / 2, \infty}$ (except, of course, for the sufficiently large number of derivatives in the arguments of Coifman-Meyer using (1.9) and which give pointwise estimates on the gradient of the kernel).

As already mentioned, we want to find some bilinear analog of the integral condition (1.3) even for non-convolution operators, but it is instructive to see what such a condition could be in the case of multipliers.

If we again consider $m$ as a Fourier multiplier in $\mathbb{R}^{2 n}$, then (1.10) is just Hörmander's condition, and we have for the kernel of the corresponding linear operator in $\mathbb{R}^{2 n}$ for any $y, z \in \mathbb{R}^{n}$

$$
\int_{\left|y-y^{\prime}\right|+\left|z-z^{\prime}\right| \leq c\left|x_{1}-y\right|+\left|x_{2}-z\right|}\left|K\left(x_{1}-y, x_{2}-z\right)-K\left(x_{1}-y^{\prime}, x_{2}-z^{\prime}\right)\right| d x_{1} d x_{2} \leq C .
$$

When $z=z^{\prime}$ and performing a simple change of variables, the above can be written as

$$
\begin{gathered}
\int_{\left|y-y^{\prime}\right| \leq c\left|x_{1}-y\right|+\left|x_{2}-z\right|}\left|K\left(x_{1}-y, x_{1}-\left(x_{1}-x_{2}+z\right)\right)-K\left(x_{1}-y^{\prime}, x_{1}-\left(x_{1}-x_{2}+z\right)\right)\right| d x_{2} d x_{1} \\
=\int_{\left|y-y^{\prime}\right| \leq c\left|x_{1}-y\right|+\left|x_{1}-u\right|}\left|K\left(x_{1}-y, x_{1}-u\right)-K\left(x_{1}-y^{\prime}, x_{1}-u\right)\right| d u d x_{1} \leq C .
\end{gathered}
$$


In a more geometric form (and considering a smaller region of integration) we can essentially state the above in the form

$$
\sup \sup _{y \in Q} \int_{\mathbb{R}^{n}} \int_{\mathbb{R}^{n} \backslash Q^{*}}\left|K(x-y, x-u)-K\left(x-y_{Q}, x-u\right)\right| d x d u \leq C,
$$

and similarly in the other variable,

$$
\sup _{Q} \sup _{y \in Q} \int_{\mathbb{R}^{n}} \int_{\mathbb{R}^{n} \backslash Q^{*}}\left|K(x-u, x-z)-K\left(x-u, x-y_{Q}\right)\right| d x d u \leq C .
$$

In the non-convolution case of interest to us, conditions (1.12) and (1.13) would become

$$
\sup _{Q} \sup _{y \in Q} \int_{\mathbb{R}^{n}} \int_{\mathbb{R}^{n} \backslash Q^{*}}\left|K(x, y, u)-K\left(x, y_{Q}, u\right)\right| d x d u \leq C
$$

and

$$
\sup _{Q} \sup _{z \in Q} \int_{\mathbb{R}^{n}} \int_{\mathbb{R}^{n} \backslash Q^{*}}\left|K(x, u, z)-K\left(x, u, z_{Q}\right)\right| d x d u \leq C .
$$

Unfortunately these conditions do not seem to be enough to show the end-point estimate we are looking for. This is not surprising since, as observed, in the convolution case they are implied by Tomita's condition (1.10), which does not seem to be even enough for boundedness for any $r \leq 1$.

We find interesting that a small modification of (1.14) and 1.15) (moving the $\sup _{y \in Q}$ inside the integral), together with a closely related new integral condition, suffices. Moreover we will show that the conditions are implied by a very general Dini-type one, hence showing that they are weaker than all others considered in the literature so far.

\section{THE NEW REgULARITY CONDITIONS}

Definition 2.1. We say that the bilinear operator with kernel $K$ satisfies the bilinear geometric Hörmander conditions (BGHC) if there exists a fixed constant $B$ such that and for any two families of disjoint dyadic cubes $D_{1}$ and $D_{2}$,

$$
\begin{aligned}
& \sup _{Q \in D_{1}} \int_{\mathbb{R}^{n}} \sup _{y \in Q} \int_{\mathbb{R}^{n} \backslash\left(\cup_{R \in D_{1}} R^{*}\right)}\left|K(x, y, z)-K\left(x, y_{Q}, z\right)\right| d x d z \leq B, \\
& \sup _{P \in D_{2}} \int_{\mathbb{R}^{n}} \sup _{z \in P} \int_{\mathbb{R}^{n} \backslash\left(\cup_{S \in D_{2}} S^{*}\right)}\left|K(x, y, z)-K\left(x, y, z_{P}\right)\right| d x d y \leq B,
\end{aligned}
$$

and

$$
\begin{gathered}
\sum_{(P, Q) \in D_{1} \times D_{2}}|P||Q| \sup _{(y, z) \in P \times Q} \int_{\mathbb{R}^{n} \backslash\left(\cup_{R \in D_{1}} R^{*}\right) \cup\left(\cup_{S \in D_{2}} S^{*}\right)}\left|K(x, y, z)-K\left(x, y_{P}, z_{Q}\right)\right| d x \\
\leq B\left(\left|\cup_{P \in D_{1}} P\right|+\left|\cup_{Q \in D_{2}} Q\right|\right) .
\end{gathered}
$$

We want to make some further remarks about these conditions. Write $\Omega_{1}=$ $\cup_{Q \in D_{1}} Q, \Omega_{1}^{*}=\cup_{Q \in D_{1}} Q^{*}, \Omega_{2}=\cup_{P \in D_{2}} P$, and $\Omega_{2}^{*}=\cup_{P \in D_{1}} P^{*}$. First, note that for 
any $y^{\prime}, y^{\prime \prime} \in Q$,

$$
\begin{gathered}
\sup _{y \in Q} \int_{\mathbb{R}^{n} \backslash \Omega_{1}^{*}}\left|K(x, y, z)-K\left(x, y^{\prime}, z\right)\right| d x \\
\lesssim \sup _{y \in Q} \int_{\mathbb{R}^{n} \backslash \Omega_{1}^{*}}\left|K(x, y, z)-K\left(x, y^{\prime \prime}, z\right)\right| d x+\int_{\mathbb{R}^{n} \backslash \Omega_{1}^{*}}\left|K\left(x, y^{\prime}, z\right)-K\left(x, y^{\prime \prime}, z\right)\right| d x \\
\lesssim \sup _{y \in Q} \int_{\mathbb{R}^{n} \backslash\left(\Omega_{1}^{*}\right.}\left|K(x, y, z)-K\left(x, y^{\prime \prime}, z\right)\right| d x,
\end{gathered}
$$

so the inner supremum and integral in (2.1) can be replaced by either

or

$$
\sup _{y^{\prime} \in Q} \sup _{y \in Q} \int_{\mathbb{R}^{n} \backslash \Omega_{1}^{*}}\left|K(x, y, z)-K\left(x, y^{\prime}, z\right)\right| d x
$$

$$
\inf _{y^{\prime} \in Q} \sup _{y \in Q} \int_{\mathbb{R}^{n} \backslash \Omega_{1}^{*}}\left|K(x, y, z)-K\left(x, y^{\prime}, z\right)\right| d x .
$$

Similarly with (2.2), where it is also equivalent to replace the supremum and integral in (2.3) by

or

$$
\sup _{(\alpha, \beta) \in P \times Q} \sup _{(y, z) \in P \times Q} \int_{\mathbb{R}^{n} \backslash\left(\Omega_{1}^{*} \cup \Omega_{2}^{*}\right)}|K(x, y, z)-K(x, \alpha, \beta)| d x
$$

$$
\inf _{(\alpha, \beta) \in P \times Q} \sup _{(y, z) \in P \times Q} \int_{\mathbb{R}^{n} \backslash\left(\Omega_{1}^{*} \cup \Omega_{2}^{*}\right)}|K(x, y, z)-K(x, \alpha, \beta)| d x .
$$

Next, we note that (2.3) is in some sense the strongest of the three conditions, since it gives a substantial part (though not all) of (2.1) and (2.2). In fact, note that $D_{1}$ and $D_{2}$ could be the same family, so assuming that (2.3) holds,

$$
\begin{gathered}
\sum_{Q \in D_{1}}|Q| \int_{\Omega_{1}} \sup _{y \in Q} \int_{\mathbb{R}^{n} \backslash \Omega_{1}^{*}}\left|K(x, y, z)-K\left(x, y_{Q}, z\right)\right| d x d z \\
\leq \sum_{Q \in D_{1}}|Q| \sum_{Q^{\prime} \in D_{1}}\left|Q^{\prime}\right| \sup _{z \in Q^{\prime}} \sup _{y \in Q} \int_{\mathbb{R}^{n} \backslash \Omega_{1}^{*}}\left|K(x, y, z)-K\left(x, y_{Q}, z\right)\right| d x \\
\leq \sum_{\left(Q, Q^{\prime}\right) \in D_{1} \times D_{1}}|Q|\left|Q^{\prime}\right| \sup _{(\alpha, \beta) \in Q \times Q^{\prime}} \sup _{(y, z) \in Q \times Q^{\prime}} \int_{\mathbb{R}^{n} \backslash\left(\Omega_{1}^{*} \cup \Omega_{1}^{*}\right)}|K(x, y, z)-K(x, \alpha, \beta)| d x \\
\leq B\left(\left|\cup_{Q \in D_{1}} Q\right|+\left|\cup_{Q^{\prime} \in D_{1}} Q^{\prime}\right|\right) \leq 2 B \sum_{Q \in D_{1}}|Q| .
\end{gathered}
$$

So, given (2.3), the condition (2.1) could be replaced with the weaker one

$$
\int_{\mathbb{R}^{n} \backslash \Omega_{1}} \sup _{y \in Q} \int_{\mathbb{R}^{n} \backslash \Omega_{1}^{*}}\left|K(x, y, z)-K\left(x, y_{Q}, z\right)\right| d x d z \leq B .
$$

In fact, this condition and the presiding computations imply

$$
\begin{gathered}
\sum_{Q \in D_{1}}|Q| \int_{\mathbb{R}^{n}} \sup _{y \in Q} \int_{\mathbb{R}^{n} \backslash \Omega_{1}^{*}}\left|K(x, y, z)-K\left(x, y_{Q}, z\right)\right| d x d z \\
\leq 2 B \sum_{Q \in D_{1}}|Q|+\sum_{Q \in D_{1}}|Q| \int_{\mathbb{R}^{n} \backslash \Omega_{1}} \sup _{y \in Q} \int_{\mathbb{R}^{n} \backslash \Omega_{1}^{*}}\left|K(x, y, z)-K\left(x, y_{Q}, z\right)\right| d x d z \\
\leq 3 B \sum_{Q \in D_{1}}|Q| .
\end{gathered}
$$


By considering just one cube, we obtain

$$
\int_{\mathbb{R}^{n}} \sup _{y \in Q} \int_{\mathbb{R}^{n} \backslash Q^{*}}\left|K(x, y, z)-K\left(x, y_{Q}, z\right)\right| d x d z \lesssim B
$$

which clearly gives (2.1). Similarly with (2.2).

We now show that the BGHC are implied by a Dini-type one. The following condition is essentially the one considered by Maldonado and Naibo [19], except that we do not require the function $\Phi$ involved to be convex.

Definition 2.2. Let $\Phi$ be increasing and such that

$$
\int_{0}^{1} \Phi(t) \frac{d t}{t}<\infty
$$

We say that $K$ satisfies a bilinear Dini-type condition if

$$
\left|K(x, y, z)-K\left(x, y^{\prime}, z^{\prime}\right)\right| \leq \frac{C}{(|x-y|+|x-z|)^{2 n}} \Phi\left(\frac{\left|y-y^{\prime}\right|+\left|z-z^{\prime}\right|}{|x-y|+|x-z|}\right)
$$

whenever $\left|y-y^{\prime}\right| \leq \frac{1}{2}|x-y|$ and $\left|z-z^{\prime}\right| \leq \frac{1}{2}|x-z|$.

By taking $\Phi(t)=t^{\epsilon}$ it follows that the bilinear Lipschitz regularity conditions imply the Dini-type one. We now show that the BGHC is actually weaker.

Proposition 2.3. The Dini-type condition implies the BGHC.

Proof. We first show that the Dini-type condition implies

$$
\int_{\mathbb{R}^{n}} \sup _{y \in Q} \int_{\mathbb{R}^{n} \backslash \Omega_{1}^{*}}\left|K(x, y, z)-K\left(x, y_{Q}, z\right)\right| d x d z \leq C
$$

for some $C>0$. Indeed,

$$
\begin{gathered}
\int_{\mathbb{R}^{n}} \sup _{y \in Q} \int_{\mathbb{R}^{n} \backslash \Omega_{1}^{*}}\left|K(x, y, z)-K\left(x, y_{Q}, z\right)\right| d x d z \\
\lesssim \int_{\mathbb{R}^{n}} \sup _{y \in Q} \int_{\mathbb{R}^{n} \backslash Q^{*}} \frac{1}{(|x-y|+|x-z|)^{2 n}} \Phi\left(\frac{\left|y-y_{Q}\right|}{|x-y|+|x-z|}\right) d x d z \\
\lesssim \int_{\mathbb{R}^{n}} \int_{\mathbb{R}^{n} \backslash Q^{*}} \frac{1}{\left(\left|x-y_{Q}\right|+|x-z|\right)^{2 n}} \Phi\left(\frac{\sqrt{n} \ell(Q)}{\left|x-y_{Q}\right|}\right) d x d z \\
\lesssim \int_{\mathbb{R}^{n} \backslash Q^{*}} \frac{1}{\left|x-y_{Q}\right|^{n}} \Phi\left(\frac{\sqrt{n} \ell(Q)}{\left|x-y_{Q}\right|}\right) d x \\
\lesssim \int_{|x|>c_{n} \ell(Q)} \frac{1}{|x|^{n}} \Phi\left(\frac{c_{n} \ell(Q)}{|x|}\right) d x<\infty .
\end{gathered}
$$

By symmetry, the proof of (2.2) is identical.

We now prove (2.3). Fix $P$ and $Q$. Then

$$
\begin{gathered}
\sup _{(y, z) \in P \times Q} \int_{\mathbb{R}^{n} \backslash\left(\Omega_{1}^{*} \cup \Omega_{2}^{*}\right)}\left|K(x, y, z)-K\left(x, y_{P}, z_{Q}\right)\right| d x \\
\lesssim \sup _{(y, z) \in P \times Q}\left(\int_{\mathbb{R}^{n} \backslash\left(P^{*} \cup Q^{*}\right)} \frac{1}{(|x-y|+|x-z|)^{2 n}} \Phi\left(\frac{\left|y-y_{P}\right|+\left|z-z_{Q}\right|}{|x-y|+|x-z|}\right) d x\right) \\
\lesssim \sup _{(y, z) \in P \times Q}\left(\int_{\mathbb{R}^{n} \backslash\left(P^{*} \cup Q^{*}\right)} \frac{1}{(|x-y|+|x-z|)^{2 n}} \Phi\left(\frac{\sqrt{n}(\ell(P)+\ell(Q))}{\left|x-y_{P}\right|+\left|x-z_{Q}\right|}\right) d x\right) \\
\lesssim \inf _{(y, z) \in P \times Q}\left(\int_{\mathbb{R}^{n} \backslash\left(P^{*} \cup Q^{*}\right)} \frac{1}{(|x-y|+|x-z|)^{2 n}} \Phi\left(\frac{\sqrt{n}(\ell(P)+\ell(Q))}{\left|x-y_{P}\right|+\left|x-z_{Q}\right|}\right) d x\right) .
\end{gathered}
$$


Then,

$$
\begin{gathered}
\sum_{(P, Q) \in D_{1} \times D_{2}}|P||Q| \sup _{(y, z) \in P \times Q} \int_{\mathbb{R}^{n} \backslash\left(\Omega_{1}^{*} \cup \Omega_{2}^{*}\right)}\left|K(x, y, z)-K\left(x, y_{P}, z_{Q}\right)\right| d x \\
\underset{(P, Q) \in D_{1} \times D_{2}}{\lesssim \sum_{(y, z) \in P \times Q}}\left(\int_{\mathbb{R}^{n} \backslash\left(P^{*} \cup Q^{*}\right)} \frac{1}{(|x-y|+|x-z|)^{2 n}} \Phi\left(\frac{\sqrt{n}(\ell(P)+\ell(Q))}{\left|x-y_{P}\right|+\left|x-z_{Q}\right|}\right) d x\right) \\
\lesssim \sum_{(P, Q) \in D_{1} \times D_{2}} \int_{P} \int_{Q} \int_{\mathbb{R}^{n} \backslash\left(P^{*} \cup Q^{*}\right)} \frac{1}{(|x-y|+|x-z|)^{2 n}} \Phi\left(\frac{\sqrt{n}(\ell(P)+\ell(Q))}{\left|x-y_{P}\right|+\left|x-z_{Q}\right|}\right) d x d y d z \\
=C\left(\sum_{(P, Q) \in D_{1} \times D_{2}: \ell(P) \leq \ell(Q)}+\sum_{(P, Q) \in D_{1} \times D_{2}: \ell(Q) \leq \ell(P)}\right)=I+I I .
\end{gathered}
$$

We estimate $I$; the other term is of course similar. Now,

$$
\begin{gathered}
I \lesssim \sum_{(P, Q) \in D_{1} \times D_{2}} \int_{P} \int_{Q} \int_{\mathbb{R}^{n} \backslash\left(P^{*} \cup Q^{*}\right)} \frac{1}{(|x-y|+|x-z|)^{2 n}} \\
\times \Phi\left(\frac{2 \sqrt{n} \ell(Q)}{\left|x-y_{P}\right|+\left|x-z_{Q}\right|}\right) d x d y d z \\
\lesssim \sum_{Q \in D_{2}} \int_{\mathbb{R}^{n} \backslash Q^{*}} \int_{Q} \sum_{P \in D_{1}} \int_{P} \frac{1}{(|x-y|+|x-z|)^{2 n}} \Phi\left(\frac{2 \sqrt{n} \ell(Q)}{\left|x-z_{Q}\right|}\right) d y d z d x \\
\lesssim \sum_{Q \in D_{2}} \int_{\mathbb{R}^{n} \backslash Q^{*}} \int_{Q} \int_{\mathbb{R}^{n}} \frac{1}{(|x-y|+|x-z|)^{2 n}} d y d z \Phi\left(\frac{2 \sqrt{n} \ell(Q)}{\left|x-z_{Q}\right|}\right) d x .
\end{gathered}
$$

We can estimate the innermost integral by $\frac{C}{|x-z|^{n}}$; hence

$$
\begin{aligned}
& I \lesssim \sum_{Q \in D_{2}} \int_{\mathbb{R}^{n} \backslash Q^{*}} \int_{Q} \frac{1}{|x-z|^{n}} \Phi\left(\frac{2 \sqrt{n} \ell(Q)}{\left|x-z_{Q}\right|}\right) d x d z \\
& \lesssim \sum_{Q \in D_{2}} \int_{Q} \int_{\mathbb{R}^{n} \backslash Q^{*}} \frac{1}{|x-z|^{n}} \Phi\left(\frac{2 \sqrt{n} \ell(Q)}{\left|x-z_{Q}\right|}\right) d x d z \\
& \lesssim \sum_{Q \in D_{2}} \int_{Q} \int_{\mathbb{R}^{n} \backslash Q^{*}} \frac{1}{\left|x-z_{Q}\right|^{n}} \Phi\left(\frac{2 \sqrt{n} \ell(Q)}{\left|x-z_{Q}\right|}\right) d x d z \\
& \lesssim \sum_{Q \in D_{2}}|Q| \int_{|x| \geq 1} \frac{1}{|x|^{n}} \Phi\left(\frac{1}{|x|}\right) d x \lesssim\left|\cup_{Q \in D_{2}} Q\right| \int_{0}^{1} \Phi(t) \frac{d t}{t}
\end{aligned}
$$

Likewise, the term $I I$ is bounded by $C\left|\cup_{P \in D_{1}} P\right|$, which completes the proof of (2.3). 


\section{The End-Point estimate}

Theorem 3.1. Let $T$ be a bilinear operator satisfying $T: L^{p_{1}} \times L^{p_{2}} \rightarrow L^{p_{3}}$ for some $1 / p_{1}+1 / p_{2}=1 / p_{3}, 1 \leq p_{1}, p_{2}<\infty$, and also the BGHC. Then, $T: L^{1} \times L^{1} \rightarrow$ $L^{1 / 2, \infty}$.

Proof. It is enough to show that

$$
\left|\left\{x \in \mathbb{R}^{n}:\left|T\left(f_{1}, f_{2}\right)(x)\right|>\lambda^{2}\right\}\right| \leq C\left(\int_{\mathbb{R}^{n}} \frac{\left|f_{1}(x)\right|}{\lambda} d x\right)^{1 / 2}\left(\int_{\mathbb{R}^{n}} \frac{\left|f_{2}(x)\right|}{\lambda} d x\right)^{1 / 2}
$$

for all $f_{1}, f_{2} \in C_{c}^{\infty}\left(\mathbb{R}^{n}\right)$. Moreover, by homogeneity we may assume that $\left\|f_{1}\right\|_{1}=$ $\left\|f_{2}\right\|_{1}=1$ and prove that

$$
\left|\left\{x \in \mathbb{R}^{n}:\left|T\left(f_{1}, f_{2}\right)(x)\right|>\lambda^{2}\right\}\right| \leq \frac{C}{\lambda},
$$

with constant $C$ independent of $\lambda$.

Fix $\lambda>0$. For $f_{1}$ we consider the standard Calderón-Zygmund decomposition at level $\lambda$ and obtain a collection of dyadic non-overlapping cubes $Q_{1, k}$ that satisfy

$$
\lambda<\frac{1}{\left|Q_{1, k}\right|} \int_{Q_{1, k}}\left|f_{1}(x)\right| d x \leq 2^{n} \lambda .
$$

If we set $\Omega_{1}=\bigcup_{k} Q_{1, k}$, then

$$
\left|\Omega_{1}\right| \leq \frac{C}{\lambda}
$$

and

$$
\left|f_{1}(x)\right| \leq \lambda \quad \text { a.e. } x \in \mathbb{R}^{n} \backslash \Omega_{1} .
$$

As usual, we write $f_{1}=g_{1}+b_{1}$, where $g_{1}$ is defined by

$$
g_{1}(x)= \begin{cases}f_{1}(x), & x \in \mathbb{R}^{n} \backslash \Omega_{1} \\ f_{Q_{1, k}}, & x \in Q_{1, k}\end{cases}
$$

and where $f_{Q}=\frac{1}{|Q|} \int_{Q} f$. The "good" function $g_{1}$ satisfies, for any $s \geq 1$,

$$
\left\|g_{1}\right\|_{s} \leq C \lambda^{1 / s^{\prime}}\left\|f_{1}\right\|_{1}^{\frac{1}{2 s^{\prime}}+\frac{1}{s}}\left\|f_{2}\right\|_{1}^{-\frac{1}{2 s^{\prime}}}=C \lambda^{1 / s^{\prime}},
$$

and $b_{1}$ is written as

$$
b_{1}(x)=\sum_{k} b_{1, k}(x)=\sum_{k}\left(f_{1}(x)-f_{Q_{1, k}}\right) \chi_{Q_{1, k}}(x) .
$$

We do the same for $f_{2}$. Via the Calderón-Zygmund decomposition at the same level $\lambda$, we obtain a collection of of dyadic non-overlapping cubes $Q_{2, k}$ with union $\Omega_{2}$ and analogous decomposition $f_{2}=g_{2}+b_{2}$ with the properties described for $f_{1}$. Set

$$
\Omega^{*}=\Omega_{1}^{*} \cup \Omega_{2}^{*}
$$


We split the distribution set we are trying to estimate into several parts as follows:

$$
\begin{aligned}
\left|\left\{x \in \mathbb{R}^{n}:\left|T\left(f_{1}, f_{2}\right)(x)\right|>\lambda^{2}\right\}\right| & \leq\left|\left\{x \notin \Omega^{*}:\left|T\left(f_{1}, f_{2}\right)(x)\right|>\lambda^{2}\right\}\right|+\left|\Omega^{*}\right| \\
& \leq\left|\left\{x \in \mathbb{R}^{n}:\left|T\left(g_{1}, g_{2}\right)(x)\right|>\lambda^{2} / 4\right\}\right| \\
& +\left|\left\{x \in \mathbb{R}^{n} \backslash \Omega^{*}:\left|T\left(g_{1}, b_{2}\right)(x)\right|>\lambda^{2} / 4\right\}\right| \\
& +\left|\left\{x \in \mathbb{R}^{n} \backslash \Omega^{*}:\left|T\left(b_{1}, g_{2}\right)(x)\right|>\lambda^{2} / 4\right\}\right| \\
& +\left|\left\{x \in \mathbb{R}^{n} \backslash \Omega^{*}:\left|T\left(b_{1}, b_{2}\right)(x)\right|>\lambda^{2} / 4\right\}\right| \\
& +\left|\Omega^{*}\right| \\
& =\left|E_{1}\right|+\left|E_{2}\right|+\left|E_{3}\right|+\left|E_{4}\right|+\left|\Omega^{*}\right| .
\end{aligned}
$$

Clearly $\left|\Omega^{*}\right|$ is of the right size, so we only need to estimate the other sets. To estimate $\left|E_{1}\right|$, we use the fact that $T: L^{p_{1}} \times L^{p_{2}} \rightarrow L^{p_{3}}$ for some $1 / p_{1}+1 / p_{2}=1 / p_{3}$ to obtain

$$
\begin{gathered}
\left|E_{1}\right| \leq \frac{C}{\lambda^{p_{3}}} \int_{\mathbb{R}^{n}}\left|T\left(g_{1}, g_{2}\right)(x)\right|^{p_{3}} d x \leq \frac{C}{\lambda^{2 p_{3}}}\left\|g_{1}\right\|_{p_{1}}^{p_{3}}\left\|g_{2}\right\|_{p_{2}}^{p_{3}} \\
\leq \frac{C}{\lambda^{2 p_{3}}} \lambda^{p_{3}\left(1-1 / p_{1}\right)} \lambda^{p_{3}\left(1-1 / p_{2}\right)}=\frac{C}{\lambda} .
\end{gathered}
$$

By symmetry, the study of $\left|E_{2}\right|$ and $\left|E_{3}\right|$ are similar, so we only consider $\left|E_{2}\right|$. We have

$$
\begin{aligned}
\left|E_{2}\right| & \leq \frac{C}{\lambda^{2}} \int_{\mathbb{R}^{n} \backslash \Omega^{*}} \sum_{k}\left|T\left(g_{1}, b_{2, k}\right)(x)\right| d x \\
& \leq \frac{C}{\lambda^{2}} \sum_{k} \int_{\mathbb{R}^{n} \backslash \Omega^{*}}\left|\int_{\mathbb{R}^{n}} \int_{Q_{2, k}} K(x, y, z) g_{1}(y) b_{2, k}(z) d z d y\right| d x \\
& \leq \frac{C}{\lambda^{2}} \sum_{k} \int_{\mathbb{R}^{n} \backslash \Omega_{2}^{*}} \mid \int_{\mathbb{R}^{n}} g_{1}(y) \int_{Q_{2, k}}\left(K(x, y, z)-K\left(x, y, z_{Q_{2, k}}\right)\right) \\
& \left.\leq \frac{C}{\lambda^{2}} \sum_{k} \int_{\mathbb{R}^{n}}\left|g_{1}(y)\right| \int_{Q_{2, k}}\left|b_{2, k}(z)\right| \int_{\mathbb{R}^{n} \backslash \Omega^{*}}|K(z) d z d y| d x, z\right)-K\left(x, y, z_{Q_{2, k}}\right) \mid d x d z d y \\
& \leq \frac{C}{\lambda^{2}} \sum_{k} \int_{\mathbb{R}^{n}}\left(\left|g_{1}(y)\right| \int_{Q_{2, k}}\left|b_{2, k}(z)\right| d z\right. \\
& \leq \frac{C}{\lambda} \int_{\mathbb{R}^{n}}\left|g_{1}(y)\right| \sum_{k}\left|Q_{2, k}\right| \sup _{z \in Q_{2, k}} \int_{\mathbb{R}^{n} \backslash \Omega^{*}}\left|K(x, y, z)-K\left(x, y, z_{Q_{2, k}}\right)\right| d x d y \\
& \leq \frac{C}{\lambda}\left\|g_{1}\right\|_{\infty} \int_{\mathbb{R}^{n}} \sum_{k}\left|Q_{2, k}\right| \sup _{z \in Q_{2, k}} \int_{\mathbb{R}^{n} \backslash \Omega^{*}}\left|K(x, y, z)-K\left(x, y, z_{Q_{2, k}}\right)\right| d x d y \\
& \left.\left.\leq C\left|\Omega_{2}\right| \leq \frac{C}{\lambda}, \quad \mid K, y, z\right)-K\left(x, y, z_{Q_{2, k}}\right) \mid d x\right) d y \\
&
\end{aligned}
$$

where we have used condition (2.2). 
It only remains to estimate

$$
\left|E_{4}\right|=\left|\left\{x \in \mathbb{R}^{n} \backslash \Omega:\left|T\left(b_{1}, b_{2}\right)(x)\right|>\lambda^{2} / 4\right\}\right| .
$$

Writing

$$
T\left(b_{1}, b_{2}\right)=\sum_{l, k} T\left(b_{1, l}, b_{2, k}\right)
$$

we have

$$
\begin{aligned}
\left|E_{4}\right| & \leq \frac{C}{\lambda^{2}} \sum_{l, k} \int_{\mathbb{R}^{n} \backslash \Omega^{*}}\left|T\left(b_{1, l}, b_{2, k}\right)(x)\right| d x \\
& \leq \frac{C}{\lambda^{2}} \sum_{l, k} \int_{\mathbb{R}^{n} \backslash \Omega^{*}}\left|\int_{Q_{1, l}} \int_{Q_{2, k}} K(x, y, z) b_{1, l}(y) b_{2, k}(z) d z d y\right| d x .
\end{aligned}
$$

Fix one of these $Q_{1, l}$ and $Q_{2, k}$ :

$$
\int_{Q_{1, l}} \int_{Q_{2, k}} b_{1, l}(y) b_{2, k}(z) d z d y=0
$$

we have

$$
\begin{aligned}
& \int_{\mathbb{R}^{n} \backslash \Omega^{*}}\left|\int_{Q_{1, l}} \int_{Q_{2, k}} K(x, y, z) b_{2, k}(z) b_{1, l}(y) d z d y\right| d x \\
&= \int_{\mathbb{R}^{n} \backslash \Omega^{*}}\left|\int_{Q_{1, l}} \int_{Q_{2, k}}\left(K(x, y, z)-K\left(x, y_{Q_{1, l}}, z_{Q_{2, k}}\right)\right) b_{2, k}(z) b_{1, l}(y) d z d y\right| d x \\
& \leq \int_{\mathbb{R}^{n} \backslash \Omega^{*}} \int_{Q_{1, l}} \int_{Q_{2, k}}\left|K(x, y, z)-K\left(x, y_{Q_{1, l}}, z_{Q_{2, k}}\right)\right| d x\left|b_{2, k}(z)\right|\left|b_{1, l}(y)\right| d z d y \\
&= \int_{Q_{1, l}} \int_{Q_{2, k}} \int_{\mathbb{R}^{n} \backslash \Omega^{*}}\left|K(x, y, z)-K\left(x, y_{Q_{1, l}}, z_{Q_{2, k}}\right)\right| d x\left|b_{2, k}(z)\right|\left|b_{1, l}(y)\right| d z d y \\
& \leq \int_{Q_{1, l}} \int_{Q_{2, k}}\left|b_{2, k}(z)\right|\left|b_{1, l}(y)\right| d z d y \\
& \times \sup _{(y, z) \in Q_{1, l} \times Q_{2, k}} \int_{\mathbb{R}^{n} \backslash \Omega^{*}}\left|K(x, y, z)-K\left(x, y_{Q_{1, l}}, z_{Q_{2, k}}\right)\right| d x \\
& \leq C \lambda^{2}\left|Q_{1, l}\right|\left|Q_{2, k}\right| \\
& \sup _{(y, z) \in Q_{1, l} \times Q_{2, k}} \int_{\mathbb{R}^{n} \backslash \Omega^{*}}\left|K(x, y, z)-K\left(x, y Q_{1, l}, z_{Q_{2, k}}\right)\right| d x .
\end{aligned}
$$

Finally,

$$
\begin{gathered}
\left|E_{4}\right| \leq C \sum_{l, k}\left|Q_{1, l}\right|\left|Q_{2, k}\right| \sup _{(y, z) \in Q_{1, l} \times Q} \int_{\mathbb{R}^{n} \backslash \Omega^{*}}\left|K(x, y, z)-K\left(x, y_{Q_{1, l}}, z_{Q_{2, k}}\right)\right| d x \\
\leq C\left(\left|\cup_{l} Q_{1, l}\right|+\left|\cup_{k} Q_{2, k}\right|\right)=c\left(\left|\Omega_{1}\right|+\left|\Omega_{2}\right|\right) \leq \frac{C}{\lambda}
\end{gathered}
$$

because of condition (2.3). 


\section{ACKNowledGement}

The authors would like to thank the referee for valuable suggestions.

\section{REFERENCES}

[1] A. P. Calderon and A. Zygmund, On the existence of certain singular integrals, Acta Math. 88 (1952), 85-139. MR0052553 (14,637f)

[2] R. R. Coifman and Yves Meyer, On commutators of singular integrals and bilinear singular integrals, Trans. Amer. Math. Soc. 212 (1975), 315-331. MR0380244 (52 \#1144)

[3] R. Coifman and Y. Meyer, Commutateurs d'intégrales singulières et opérateurs multilinéaires, Ann. Inst. Fourier (Grenoble) 28 (1978), no. 3, xi, 177-202 (French, with English summary). MR511821 (80a:47076)

[4] Ronald R. Coifman and Yves Meyer, Au delà des opérateurs pseudo-différentiels, Astérisque, vol. 57, Société Mathématique de France, Paris, 1978 (French). With an English summary. MR.518170 (81b:47061)

[5] Michael Christ, Weak type (1,1) bounds for rough operators, Ann. of Math. (2) 128 (1988), no. 1, 19-42, DOI 10.2307/1971461. MR951506 (89m:42013)

[6] Michael Christ and Jean-Lin Journé, Polynomial growth estimates for multilinear singular integral operators, Acta Math. 159 (1987), no. 1-2, 51-80, DOI 10.1007/BF02392554. MR 906525 (89a:42024)

[7] Michael Christ and José Luis Rubio de Francia, Weak type $(1,1)$ bounds for rough operators. II, Invent. Math. 93 (1988), no. 1, 225-237, DOI 10.1007/BF01393693. MR.943929 (90d:42021)

[8] Loukas Grafakos, Akihiko Miyachi, and Naohito Tomita, On multilinear Fourier multipliers of limited smoothness, Canad. J. Math. 65 (2013), no. 2, 299-330, DOI 10.4153/CJM-2012025-9. MR3028565

[9] Loukas Grafakos and Zengyan Si, The Hörmander multiplier theorem for multilinear operators, J. Reine Angew. Math. 668 (2012), 133-147. MR.2948874

[10] Loukas Grafakos and Rodolfo H. Torres, On multilinear singular integrals of CalderónZygmund type, Proceedings of the 6th International Conference on Harmonic Analysis and Partial Differential Equations (El Escorial, 2000), 2002, pp. 57-91, DOI 10.5565/PUBLMAT_Esco02_04. MR1964816 (2004c:42031)

[11] Loukas Grafakos and Rodolfo H. Torres, Multilinear Calderón-Zygmund theory, Adv. Math. 165 (2002), no. 1, 124-164, DOI 10.1006/aima.2001.2028. MR.1880324 (2002j:42029)

[12] Loukas Grafakos and Rodolfo H. Torres, Maximal operator and weighted norm inequalities for multilinear singular integrals, Indiana Univ. Math. J. 51 (2002), no. 5, 1261-1276, DOI 10.1512/iumj.2002.51.2114. MR.1947875 (2003j:42020)

[13] S. Hofmann, Featured Review of Singular integral operators with rough convolution kernels, J. Amer. Math. Soc. 9 (1996), no. 1, 95-105, by A. Seeger, Math. Rev. MR1317232 (96f:42021)

[14] Lars Hörmander, Estimates for translation invariant operators in $L^{p}$ spaces, Acta Math. 104 (1960), 93-140. MR0121655 (22 \#12389)

[15] Carlos E. Kenig and Elias M. Stein, Multilinear estimates and fractional integration, Math. Res. Lett. 6 (1999), no. 1, 1-15. MR.1682725 (2000k:42023a)

[16] Michael Lacey and Christoph Thiele, $L^{p}$ estimates on the bilinear Hilbert transform for $2<p<\infty$, Ann. of Math. (2) 146 (1997), no. 3, 693-724, DOI 10.2307/2952458. MR 1491450 (99b:42014)

[17] Michael Lacey and Christoph Thiele, On Calderón's conjecture, Ann. of Math. (2) 149 (1999), no. 2, 475-496, DOI 10.2307/120971. MR1689336 (2000d:42003)

[18] Andrei K. Lerner, Sheldy Ombrosi, Carlos Pérez, Rodolfo H. Torres, and Rodrigo TrujilloGonzález, New maximal functions and multiple weights for the multilinear CalderónZygmund theory, Adv. Math. 220 (2009), no. 4, 1222-1264, DOI 10.1016/j.aim.2008.10.014. MR2483720 (2010f:42024)

[19] Diego Maldonado and Virginia Naibo, Weighted norm inequalities for paraproducts and bilinear pseudodifferential operators with mild regularity, J. Fourier Anal. Appl. 15 (2009), no. 2, 218-261, DOI 10.1007/s00041-008-9029-x. MR2500923 (2010b:42027) 
[20] José María Martell, Carlos Pérez, and Rodrigo Trujillo-González, Lack of natural weighted estimates for some singular integral operators, Trans. Amer. Math. Soc. 357 (2005), no. 1, 385-396 (electronic), DOI 10.1090/S0002-9947-04-03510-X. MR2098100 (2005f:42036)

[21] S. G. Mihlin, On the multipliers of Fourier integrals, Dokl. Akad. Nauk SSSR (N.S.) 109 (1956), 701-703 (Russian). MR0080799 (18,304a)

[22] Carlos Pérez and Rodolfo H. Torres, Sharp maximal function estimates for multilinear singular integrals, Harmonic analysis at Mount Holyoke (South Hadley, MA, 2001), Contemp. Math., vol. 320, Amer. Math. Soc., Providence, RI, 2003, pp. 323-331, DOI 10.1090/conm/320/05615. MR1979948 (2004e:42022)

[23] Andreas Seeger, Singular integral operators with rough convolution kernels, J. Amer. Math. Soc. 9 (1996), no. 1, 95-105, DOI 10.1090/S0894-0347-96-00185-3. MR1317232(96f:42021)

[24] Naohito Tomita, A Hörmander type multiplier theorem for multilinear operators, J. Funct. Anal. 259 (2010), no. 8, 2028-2044, DOI 10.1016/j.jfa.2010.06.010. MR2671120 (2011h:42013)

Departamento de Análisis Matemático, Facultad de Matemáticas, Universidad de Sevilla, 41080 Sevilla, Spain

E-mail address: carlosperez@us.es

Department of Mathematics, University of Kansas, 405 Snow Hall, 1460 Jayhawk Boulevard, LaWrence, Kansas 66045-7523

E-mail address: torres@math.ku.edu 